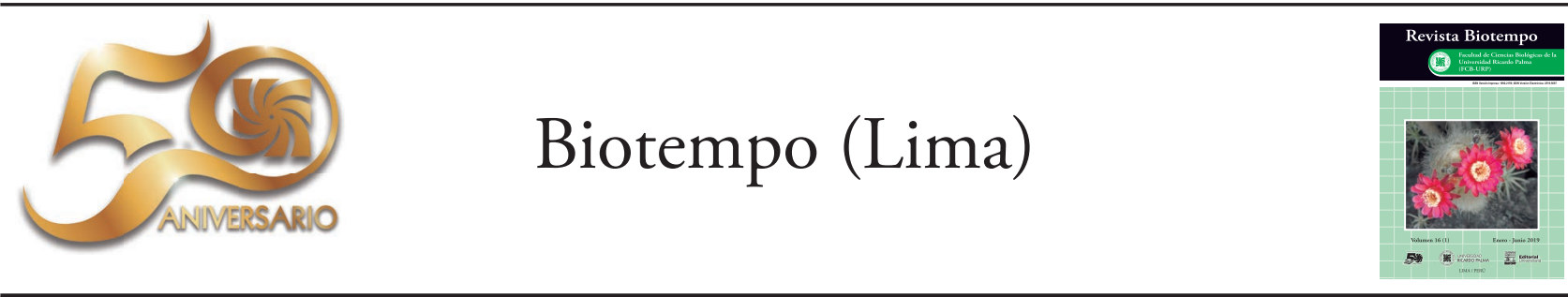

ORIGINAL ARTICLE / ARTÍCULO ORIGINAL

RAIMONDICERAS RAIMONDII (GABB, 1877) (AMMONOIDEA): PRELIMINARY TAXONOMIC STUDY

\title{
RAIMONDICERAS RAIMONDII (GABB, 1877) (AMMONOIDEA): ESTUDIO TAXONÓMICO PRELIMINAR
}

\author{
${ }^{1}$ Vera Alleman Haeghebaert
}

1 Facultad de Ciencias Biológicas/ Museo de Historia Natural "Vera Alleman Haeghebaert", Universidad Ricardo Palma. E-mail: vmealleman@yahoo.es

Author for correspondence: vmealleman@yahoo.es

\begin{abstract}
A reference taxonomic collection deposited in the Department of Paleontology of the Museum of Natural History "Vera Alleman Haeghebaert" of Ricardo Palma University consists of the identification, registration and description of seventeen specimens of the genus Raimondiceras Spath 1924, of which twelve specimens were attributed to the species Raimondiceras raimondii (Gabb, 1877). The initial taxonomic study was based on the publications of William More Gabb and Carlos Lissón. In addition, the identification key of the Ammonoidea species of the Morro Solar Group, Valanginian-Hauterivian de Lima, Peru was used. Having lost all the "types", original specimens of the species, we intend to start the complementary and updated description of the species. The place where Antonio Raimondi and Carlos Lissón obtained the first specimens of $R$. raimondii was specified: the La Herradura Formation outcrops in the hills of the Morro Solar de Chorrillos, Lima. Raimondiceras raimondii was found only in the La Herradura Formation.
\end{abstract}

Keywords: Hauterivian- Perisphinctaceae - Taxonomy - Types - Valanginian

\section{RESUMEN}

De una colección taxonómica de referencia depositada en el Departamento de Paleontología del Museo de Historia Natural "Vera Alleman Haeghebaert" de la Universidad Ricardo Palma, Lima, Perú, se identificó, registró y describió diez y siete ejemplares del género Raimondiceras Spath, 1924, de los cuales doce ejemplares fueron atribuidos a la especie Raimondiceras raimondii (Gabb, 1877). El estudio taxonómico inicial se apoyó sobre las publicaciones de William More Gabb y de Carlos Lissón. Además, se utilizó la clave de identificación de las especies de Ammonoidea del Grupo Morro Solar, Valanginiano-Hauteriviano de Lima, Perú. Habiendo desaparecidos todos los “tipos", ejemplares originales de la especie, se pretende iniciar la descripción complementaria y actualizada de la especie, realizando una nueva colección de material como base de reconstrucción de una nueva colección de tipos de esta especie. Se precisó el lugar donde Antonio Raimondi y Carlos Lissón consiguieron los primeros ejemplares de $R$. raimondii, que fue La Formación La Herradura 
que aflora en los cerros del Morro Solar de Chorrillos, Lima, Perú. Raimondiceras raimondii se encontró únicamente en el Miembro La Herradura.

Palabras clave: Hauteriviano - Perisphinctaceae - Taxonomia - Tipos - Valanginiano

\section{INTRODUCCIÓN}

Raimondiceras raimondii (Gabb, 1877) es una especie del género Raimondiceras Spath, 1924, siendo el género y la especie taxones peruanos (Gabb, 1877). $R$. raimondii ha sido descubierta por Antonio Raimondi y ha sido descrito por William More Gabb al final del siglo XVIII y luego Carlos Lissón completó las descripciones al inicio del siglo IXX. La determinación original de la especie se basa sobre un total de cuatro ejemplares, un original de Raimondi y tres originales recolectados por C. Lissón. Actualmente no hemos encontrado ninguno de estos ejemplares originales de la especie $R$ raimondi (Lissón, 1904; 1907). Además las descripciones e ilustraciones de esas épocas están consideradas ahora bastante obsoletas e incompletas y se necesita consolidar la validez de esta especie, en la medida de lo posible, por un estudio taxonómico actualizado respaldado por un mayor número de ejemplares, una terminología descriptiva más precisa y el apoyo de la tecnología ilustrativa e reconstructiva del siglo XX (Romero et al., 1995).

En esta ocasión, nuestra contribución consiste en la presentación de la existencia de una nueva colección registrada de $R$. raimondii recolectada en la localidad original y depositada en el Museo de Historia Natural "Vera Alleman Haeghebaert" de la Universidad Ricardo Palma, Lima, Perú. Es un primer avance de un estudio taxonómico actualizado de la especie.

\section{MATERIAL Y MÉTODOS}

El material proviene de la localidad Morro Solar, Departamento de Lima, Provincia de Lima, Distrito de Chorrillos. Contexto litoestratigráfico Grupo Morro Solar, Formación La Herradura, Miembro La Herradura. Contexto cronoestratigráfico Mesozoico, Cretáceo, Valanginiano. Sus coordenadas geográficas son longitud $77^{\circ}$ 01' 57”.7 W y Latitud 12¹1'07”.9 S (Palacios et al., 1992).

La recolección se hizo durante las prácticas de campo de las asignaturas de Paleontología y de Paleobiología de los alumnos de la Facultad de Ciencias Biológicas de la Universidad Ricardo Palma, Lima, Perú.

El estudio taxonómico inicial se apoyó sobre las publicaciones de Gabb (1877) y de Lissón (1904, 1907). Además se utilizó la clave de identificación de las especies de Ammonoidea del Grupo Morro Solar, ValanginianoHauteriviano de Lima, Perú (Alleman, 2014).

La colección dispone de una cantidad de doce ejemplares de esta especie, recolectados en la localidad típica, registrados bajo los códigos siguientes: VA011116 (Fig. 1); VA021216; VA030413; VA060413; VA070413; VA071216; VA081216 (Fig. 2); VA110413; VA340413; VA450413; VA500413 y VA720413. Dentro de la fauna asociada figuran las especies: Raimondiceras pflueckeri (Lissón, 1904) y Favrella lorensis (Lissón, 1907), actualmente en estudio (Villaseca, 2017).

Aspectos éticos: La identificación taxonómica y el registro han sido cumplidos según las normas exigidas por el Ministerio Nacional de Cultura para su incorporación como bienes muebles de la Nación.

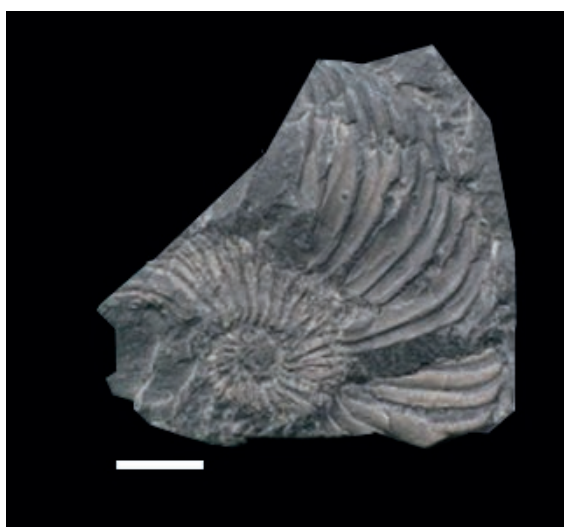

Figura 1. VA011116: Raimondiceras raimondii (Gabb, 1877). Escala $1 \mathrm{~cm}$.

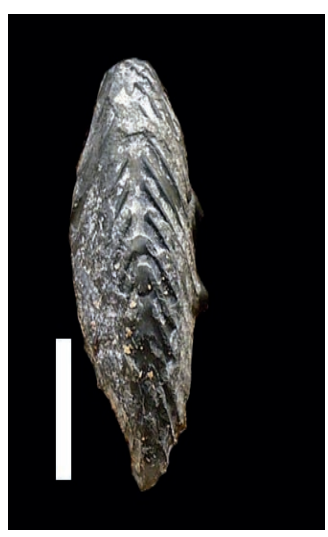

Figura 2. VA081216: Dorso de Raimondiceras raimondii (Gabb, 1877). Escala $1 \mathrm{~cm}$. 


\section{RESULTADOS Y DISCUSIÓN}

Las investigaciones de Rivera \& Alleman (1974) y de Tapia (1988) para ubicar los ejemplares originales de los fósiles peruanos y de Lima reportan que solamente un ejemplar tipo de $R$. raimondii se encontró en repositorio en la Universidad Nacional de Ingeniería, Lima, Perú. Se trata de la especie tipo UNI: T1 (2592) que corresponde a la Lám. 5, fig. 1 en Lissón (1907).

En el presente año 2019, ya no hemos podido ubicar este ejemplar tampoco.

Citando a Tapia (1988) nuevamente: "No se pudieron ubicar todos los demás ejemplares tipos de $R$. raimondii, que son los siguientes:

1) El ejemplar que corresponde a la descripción de Gabb (1877).

2) El ejemplar de las figuras 23-25 en Lissón (1904.)

3) El ejemplar de las figuras 26-28 de Lissón (1904) = Lam, 5, fig.2 (Lisson, 1907)".

Confirmamos estos datos.

Todo este material proviene exclusivamente del Miembro La Herradura de la Formación La Herradura, exactamente de la localidad de donde proviene el ejemplar recolectado por Raimondi. En consecuencia, estos son potencialmente candidatos a futuros ejemplares "tipo", susceptibles de ratificar las características de la especie o/y complementar su conocimiento.

Lamentablemente todos los individuos se fosilizaron en forma incompleta $y$, refiriéndonos a las ilustraciones de Lissón (Lissón, 1907: 41-43; Lam. V, Fig. 1,2), permanecen en un mismo rango de desarrollo mayormente juvenil. No tenemos ninguna muestra de $R$. raimondii con la región bucal y tampoco no fue ubicado, tanto por nosotros como por Lissón, ninguna muestra como la encontrada por Raimondi.

Los ejemplares identificados de la colección forman una variedad de material nuevo para estudios taxonómicos ampliados.

Se espera que la presente colección debidamente investigada ratifica y complete los datos de los autores anteriores y amplificará los conocimientos paleobiológicos de la especie.

\section{AGRADECIMIENTOS}

Agradecemos a la Universidad Ricardo Palma, así como al Instituto Peruano de Astronomía por el apoyo al presente tema de investigación.

\section{REFERENCIAS BIBLIOGRÁFICAS}

Alleman, V. 2014. Clave de Identificación de las Especies de Ammonoidea del Grupo Morro Solar, Valanginiano-Hauteriviano de Lima, Perú. Resumenes Extendidos del XVII Congreso Peruano de Geología, 12 al 15 de Octubre de 2014. Libro de Resúmenes. INGEMMET: 4 pp.

Gabb, W.M. 1877. Description of a Collection of fossils made by Dr. A. Raimondi in Perú. Journal of the Academy of Natural Sciences of Philadelphia, Serie 2, 8: 263-336.

Lissón, C. 1904 Los Tigillites del Salto del Fraile y algunos Sonneratias del Morro Solar. Contribución a la Geología de Lima y Alrededores. Imprenta Gil. Lima.

Lissón, C. 1907 Contribución a la Geología de Lima y Alrededores. Imprenta Gil. Lima.

Palacios, O.; Caldos, J. \& Churchil, V. 1992. Geología de los cuadrángulos de Lima, Lurín, Chancay y Chosica. Boletín No 43, Serie A: 1-152.

Rivera, R. \& Alleman, V. 1974. Fósiles "Tipos" conservados en el Perú. Boletin de la Sociedad Geológica del Perú (Lima), 44: 80-104.

Romero, L.; Aldana, M.; Villavicencia, E. \& Ramírez, J. 1995. Fauna y Flora fósil del Perú. Boletín 17, Serie D: Estudios Especiales. INGEMMET. $331 \mathrm{p}$.

Tapia, P. 1988. Revisión bibliográfica de los Ammonoidea (Cephalopoda, Mollusca) del Cretáceo de Lima. Tesis Bach. Biología, Universidad Ricardo Palma (inédita).

Villaseca, A. 2017. Favrella lorensis (Lisson, 1907): Fauna y flora asociadas. II Jornadas de Jóvenes Investigadores en Paleontología. Ministerio de Cultura: pp. 74-77.

Received May 23, 2019.

Accepted June 1, 2019. 